\title{
Article
}

\section{LONG-LIVED DOUBLE-BARRED GALAXIES FROM PSEUDOBULGES}

Debattista, Victor P and Shen, Juntai

Available at https://clok.uclan.ac.uk/16868/

Debattista, Victor P orcid iconORCID: 0000-0001-7902-0116 and Shen, Juntai (2007) LONG-LIVED DOUBLE-BARRED GALAXIES FROM PSEUDOBULGES. The Astrophysical Journal Letters . L127-L130. ISSN 2041-8205

It is advisable to refer to the publisher's version if you intend to cite from the work.

For more information about UCLan's research in this area go to

http://www.uclan.ac.uk/researchgroups/ and search for < name of research Group>.

For information about Research generally at UCLan please go to

http://www.uclan.ac.uk/research/

All outputs in CLoK are protected by Intellectual Property Rights law, including Copyright law. Copyright, IPR and Moral Rights for the works on this site are retained by the individual authors and/or other copyright owners. Terms and conditions for use of this material are defined in the policies page.

\section{CLoK}

Central Lancashire online Knowledge www.clok.uclan.ac.uk

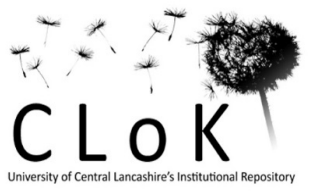




\title{
LONG-LIVED DOUBLE-BARRED GALAXIES FROM PSEUDOBULGES
}

\author{
Victor P. Debattista ${ }^{1}$ and Juntai Shen ${ }^{2}$ \\ Received 2006 August 16; accepted 2006 November 29; published 2006 December 28
}

\begin{abstract}
A large fraction of barred galaxies host secondary bars that are embedded in their large-scale primary counterparts. The evolution of such double-barred galaxies is still not well understood, partly because of a lack of realistic $N$-body models with which to study them. Here we report a new mechanism for generating such systems, namely, the presence of rotating pseudobulges. We demonstrate with high mass and force resolution collisionless $N$-body simulations that long-lived secondary bars can form spontaneously without requiring gas, contrary to previous claims. We find that secondary bars rotate faster than primary ones. The rotation is not rigid: the secondary bars pulsate, with their amplitude and pattern speed oscillating as they rotate through the primary bars. This self-consistent study supports previous work based on orbital analysis in the potential of two rigidly rotating bars. The pulsating nature of secondary bars may have important implications for understanding the central region of double-barred galaxies.

Subject headings: galaxies: bulges — galaxies: evolution — galaxies: formation -

galaxies: kinematics and dynamics — galaxies: structure — stellar dynamics

Online material: mpeg animation
\end{abstract}

\section{INTRODUCTION}

Double-barred (S2B) galaxies, consisting of a small-scale nuclear/secondary bar (B2) embedded within a large-scale primary bar (B1), have been known for over 30 years (e.g., de Vaucouleurs 1975). Erwin \& Sparke (2002) carefully compiled statistics for early-type optically barred galaxies from images by both the WIYN telescope and the Hubble Space Telescope and concluded that at least one-quarter of them are doublebarred. The facts that inner bars are also seen in near-infrared (e.g., Mulchaey et al. 1997; Laine et al. 2002), and that gaspoor SB0 galaxies (e.g., Petitpas \& Wilson 2004) often contain inner bars, indicate that most of them are stellar structures. S2B galaxies may play an important role in the formation and nurture of supermassive black holes (SMBHs). S2B galaxies have been hypothesized to be a possible mechanism for driving gas past the inner Lindblad resonance of B1s, feeding SMBHs and powering active galactic nuclei (AGNs) (Shlosman et al. 1989). S2B galaxies have also been suggested as a mechanism for forming SMBHs directly (Begelman et al. 2006).

Such fueling requires that the B2 and the B1 are dynamically decoupled. ${ }^{3}$ The random orientations of B1s and B2s in nearly face-on galaxies points to dynamical decoupling (Buta \& Crocker 1993; Friedli \& Martinet 1993) but cannot reveal how the two bars rotate. Gas or stellar kinematic evidence of decoupling is harder to obtain (Petitpas \& Wilson 2002; Schinnerer et al. 2002; Moiseev et al. 2004). Indirect evidence for decoupling was suggested by Emsellem et al. (2001) based on rotation velocity peaks inside the B2s in three S2B galaxies. Conclusive direct kinematic evidence for a decoupled B2 was obtained for NGC 2950 by Corsini et al. (2003).

An important advance in understanding S2B galaxies came from the development by Maciejewski \& Sparke (1997, 2000, hereafter MS00) of the formalism necessary for studying their orbits. They introduced the concept of loops, families of orbits

\footnotetext{
${ }^{1}$ Brooks Prize Fellow; Astronomy Department, University of Washington, Seattle, WA; debattis@astro.washington.edu

${ }^{2}$ Harlan J. Smith Fellow; McDonald Observatory, University of Texas at Austin, Austin, TX; shen@astro.as.utexas.edu

${ }^{3}$ In this context, by decoupled we mean only that $\Omega_{\mathrm{B} 2} \neq \Omega_{\mathrm{B} 1}$, where $\Omega_{\mathrm{B} 2}$ $\left(\Omega_{\mathrm{B} 1}\right)$ is the pattern speed of the B2 (B1).
}

in which particles return to the same curve, but not the same position, after the two bars return to the same relative orientation. MS00 considered two models assuming that the B2 is more rapidly rotating than the $\mathrm{B} 1$ : the $\mathrm{B} 2$ in their model 1 ended near its corotation radius, while in their model 2 it ended well inside this radius. MSO0 were unable to find loop orbits supporting the outer parts of the B2 in model 1 but succeeded in doing so in the more slowly rotating model 2 . Using hydrodynamical simulations of such slowly rotating rigid B2s, Maciejewski et al. (2002) found them to be inefficient at driving gas to small radii.

Such models are not fully self-consistent since, in general, nested bars cannot rotate rigidly through each other (Louis \& Gerhard 1988). In fact, nonsolid body rotation was hinted at by the loop orbit calculations of MS00. $N$-body simulations provide one route to more self-consistent models of S2B galaxies, but until now there existed a paucity of such models. Most numerical studies (e.g., Shlosman \& Heller 2002; Friedli \& Martinet 1993; Englmaier \& Shlosman 2004) required gas to form B2s; for example, Heller et al. (2001) formed them via viscosity-driven instabilities in nuclear gas rings, which lead to B2s rotating slower than B1s. But the presence of B2s in a large fraction of gas-poor early-type galaxies (Erwin \& Sparke 2002; Petitpas \& Wilson 2004) indicates that B2s are not an exclusively gas dynamical phenomenon. Counterrotation in stellar disks can lead to decoupled counterrotating bars (Sellwood \& Merritt 1994; Friedli 1996; Davies \& Hunter 1997), but such counterrotation is infrequent (Kuijken et al. 1996). Only Rautiainen and collaborators (Rautiainen \& Salo 1999; Rautiainen et al. 2002) have succeeded in forming long-lived B2s rotating in the same sense as the B1 in purely collisionless studies, although these B2s often had a "vaguely spiral shape." The mechanism by which the B2s formed in these simulations also remains unclear.

In light of the increasing evidence that SMBH feedback may play an important role in galaxy formation (Springel et al. 2005) and the possibility that S2B galaxies may provoke AGNs, the paucity of self-consistent $N$-body models of S2B galaxies is a major hindrance to further theoretical development. The time is ripe, therefore, to examine whether unambiguous and independently rotating nested bars can form in high-resolution collisionless simulations. Kormendy \& Kennicutt (2004) pointed out that a nuclear bar constitutes strong evidence of a pseudobulge, 

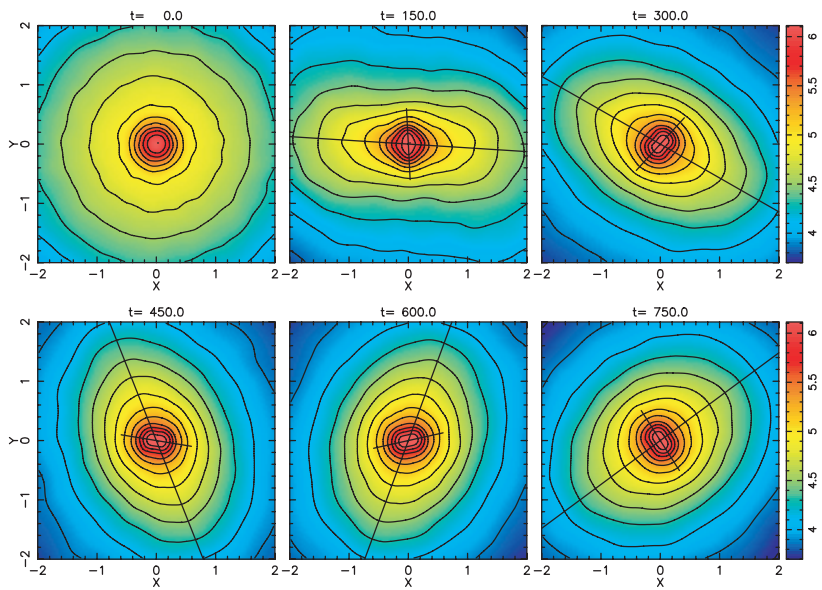

FIG. 1.-Images of total stellar distribution at various times, with isodensity contours superposed. The contours are logarithmic and separated by 0.2 dex. The heavy short and long straight lines mark the major axes of the B2 and $\mathrm{B} 1$, respectively. The surface density is obtained by smoothing every particle with an adaptive kernel (Silverman 1986). Note that 100 time units is about $1.2 \mathrm{Gyr}$, and the length unit is the scale length of the initial disk.

i.e., a bulge with a disky character. Such pseudobulges form through the secular evolution of disks, via both gas and stellar dynamical processes (see the review of Kormendy \& Kennicutt 2004). One of the main characteristics of pseudobulges is that they rotate rapidly, a property that favors them to become barunstable. In this work, we demonstrate that a rapidly rotating bulge can develop a long-lived B2 in collisionless $N$-body simulations.

\section{MODEL SETUP}

We focus on two examples of simulations that formed longlasting double-barred systems taken from a large survey of such simulations. Our high-resolution simulations consist of live disk and bulge components in a rigid halo potential. We restrict ourselves to rigid halos to afford high mass resolution in the nuclear regions, to study the complicated coevolution of the two bars without the additional evolution introduced by the halo, and to compare our models with those of MS00. We defer the study of S2B systems in live halos to a future publication. The rigid halos used in this study are all logarithmic potentials $\Phi(r)=$
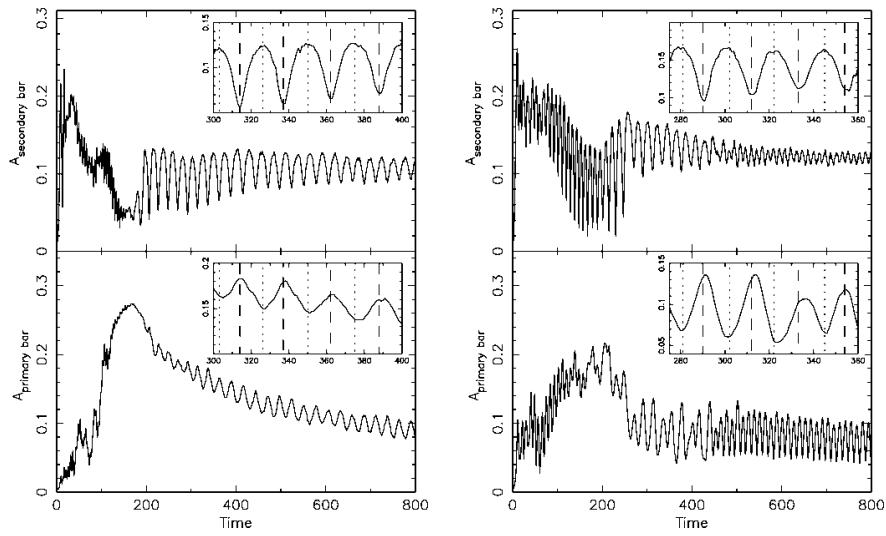

FIG. 2.-Time evolution of the bar amplitude of the B2 (top panels) and the B1 (bottom panels). In the insets, the dashed lines mark times when the two bars nearly align, while the dotted lines mark the time when they are perpendicular to each other. The beat period $\tau_{\text {beat }}=\tau_{\mathrm{B} 1} \tau_{\mathrm{B} 2} / 2\left|\tau_{\mathrm{B} 1}-\tau_{\mathrm{B} 2}\right|$. Run 1 is on the left; run 2 is on the right. $\frac{1}{2} V_{h}^{2} \ln \left(r^{2}+r_{h}^{2}\right)$. We set $V_{h}=0.6$ in both runs and $r_{h}=15$ in run 1 and $r_{h}=10$ in run 2 . Both initial disks in our simulations have exponential surface densities with scale length $R_{d}$, mass $M_{d}$, and Toomre $Q \simeq 2$. The bulge was generated using the method of Prendergast \& Tomer (1970) as described in Debattista $\&$ Sellwood (2000), where a distribution function is integrated iteratively in the global potential, until convergence. In both cases the bulge has mass $M_{b}=0.2 M_{d}$, and we used an isotropic King model distribution function. The bulge truncation radius is $0.7 R_{d}$ in run 1 and $1.0 R_{d}$ in run 2 . The bulge set up this way is nonrotating. We introduce bulge rotation by reversing the velocities of particles with negative angular momenta, which remains a valid solution of the collisionless Boltzmann equation (Lynden-Bell 1962). The bulge in run 1 is flattened by the disk potential to an edge-on projected ellipticity of $\epsilon_{b} \simeq 0.25$. The ratio $V_{p} / \bar{\sigma} \simeq 0.8$, where $V_{p}$ is the peak velocity and $\bar{\sigma}$ is the average velocity dispersion inside the half mass radius. In run 2 , the corresponding values are $\epsilon_{b} \simeq 0.38$ and $V_{p} / \bar{\sigma} \simeq 0.7$. The kinematic values relative to the oblate isotropic rotators are $\left(V_{p} / \bar{\sigma}\right)_{*} \simeq 1.3$ for run 1 and $\left(V_{p} / \bar{\sigma}\right)_{*} \simeq 0.9$ for run 2 . Thus, both pseudobulges are above or close to the locus of oblate isotropic rotators. These pseudobulges are less tangentially biased and more pressure-supported than rotationally supported pseudobulges that would form out of gas driven to small radii. Our simulations therefore probably underestimate the tendency for pseudobulges to form nuclear bars.

We use $R_{d}$ and $M_{d}$ as the units of length and mass, and the time unit is $\left(R_{d}^{3} / G M_{d}\right)^{1 / 2}$. A possible scaling to physical values is $M_{d}=2.3 \times 10^{10} M_{\odot}$ and $R_{d}=2.5 \mathrm{kpc}$, which gives a time unit of 12.3 Myr. We use a force resolution (softening) of 0.01 (corresponding to $25 \mathrm{pc}$ ). Both models had $1.2 \times 10^{6}$ equal mass particles, with $10^{6}$ in the disk. These simulations were evolved with a three-dimensional cylindrical polar grid code (Sellwood $\&$ Valluri 1997), which expands the potential in a Fourier series (which we truncated at $m=8$ ) in the cylindrical polar angle $\phi$. Forces in the radial direction are solved for by direct convolution with the Greens function, while the vertical forces are obtained by fast Fourier transform. We used grids measuring $N_{R} \times$ $N_{\phi} \times N_{z}=58 \times 64 \times 375$. The vertical spacing of the grid planes was $\delta z=0.01 R_{d}$. Time integration used a leapfrog integrator with a fixed time step $\delta t=0.04$.

\section{RESULTS}

Figure 1 gives a general view of the evolution of run 1 over 750 time units ( 9.2 Gyr in our standard scaling). A nuclear bar forms rapidly (before $t=10$ ), as the dynamical times in the inner galaxy are short. The pattern speed, $\Omega_{\mathrm{B} 2}$, of this nuclear bar is large at this stage, and it extends to nearly its corotation radius, $R_{c, \mathrm{~B} 2}$, indicating that it forms by the usual bar instability (Toomre 1981). The B1 forms at a later stage, between $t=100$ and 200. The evolution of the amplitudes of the $\mathrm{B} 1$ and $\mathrm{B} 2\left(A_{\mathrm{B} 1}\right.$ and $A_{\mathrm{B} 2}$, respectively), defined as the Fourier $m=2$ amplitude over the radial ranges $0.5 \leq R \leq 2$ and $R \leq 0.3$, is shown in the left panel of Figure 2. The B2 is strong initially, but it weakens once the B1 forms. At the same time $\Omega_{\mathrm{B} 2}$ also decreases and, since its semimajor axis does not change substantially, it no longer extends to $R_{c, \mathrm{~B} 2}$. After $t=250$ the $\mathrm{B} 2$ settles into an oscillatory steady state with $A_{\mathrm{B} 2}$ exhibiting regular oscillations. The double-barred state persists to the end of the simulation, lasting for $\sim 7$ Gyr. The B2 shows up in both the disk and bulge particles.

The B2 is stronger when the bars are perpendicular and weaker when they are parallel to each other (Fig. 2). Note that this behavior is similar to that of the gaseous nuclear ring in Rau- 

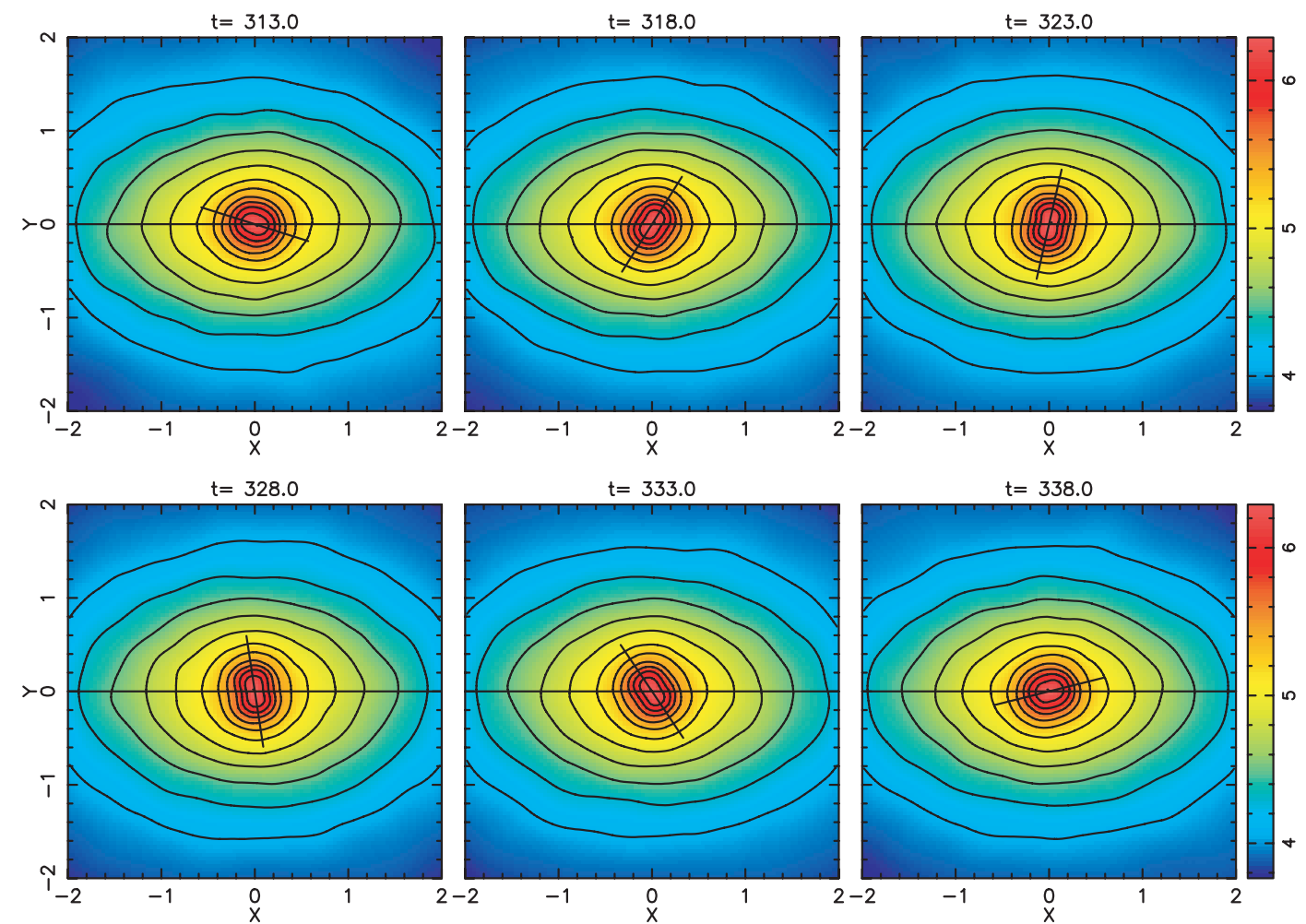

FIG. 3.-Nonuniform relative rotation of the B2 for roughly half of a period, in the corotating frame of the B1 that remains horizontal. The panels are equally spaced in time. The straight line marks the major axis of the B2. The B2 rotates faster when the two bars align than when the two bars are perpendicular. This figure is also available as an mpeg animation in the electronic edition of the Astrophysical Journal Letters.

tiainen et al. (2002, their Fig. 3) but exactly opposite to the variations of gaseous rings in Heller et al. (2001). The amplitude of the primary bar instead varies in the opposite sense with respect to the relative phase of the two bars, although the amplitude of this oscillation is smaller. $A_{\mathrm{B} 1}$ also decreases slowly after $t=250$, possibly because orbits supporting the B1 are gradually disrupted by the relatively strong inner bar. As a consequence, the oscillations in $A_{\mathrm{B} 2}$ decrease as the $\mathrm{B} 1$ weakens.

The right panel of Figure 2 shows the amplitudes evolution

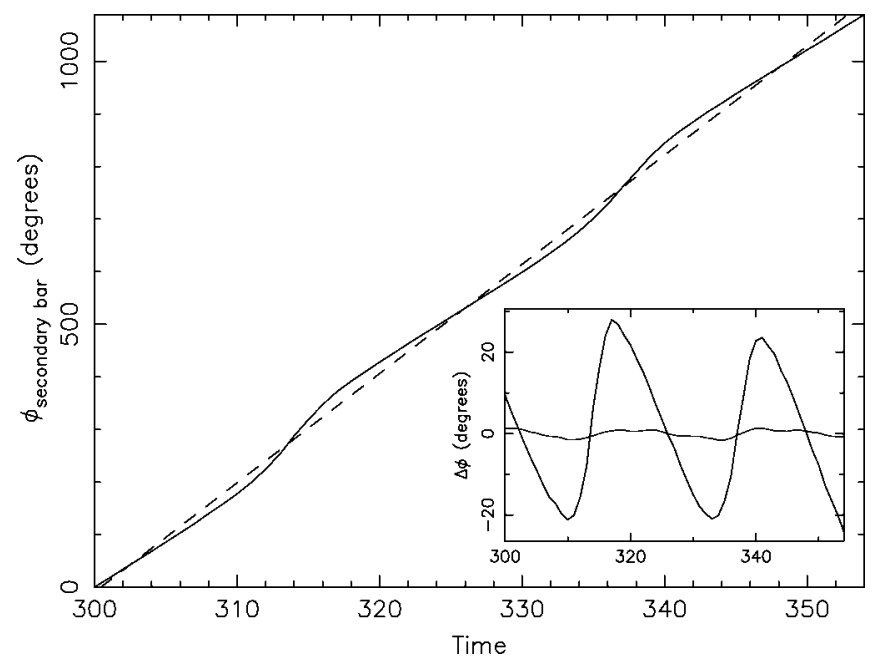

FIG. 4.-Time evolution of the phase of the B2, measured relative to $t=$ 300. The dashed straight line is the least-square fit, which gives the slope $\left\langle\Omega_{\mathrm{B} 2}\right\rangle$. The inset figure shows the phase difference, $\Delta \phi$, between the phases of the bars and $\langle\Omega\rangle t$, where $\langle\Omega\rangle$ is the pattern speed averaged over one relative rotation of the two bars; the thick line is for the B2 while the thin line is for the B1. in run 2. The main difference compared with run 1 is that the bulge is larger in run 2, allowing the $\mathrm{B} 2$ to dominate the global dynamics. Thus, the B1 oscillates more strongly than the B2. This probably represents an extreme case of the dynamical influence of a B2 on a B1.

The long-lived B2 rotates faster than the B1: between $t=$ 300 and 400 the average rotation period of the B2 in run 1 is about $\tau_{\mathrm{B} 2} \simeq 17.6$, and for the $\mathrm{B} 1 \tau_{\mathrm{B} 1} \simeq 27.8$. The faster $\mathrm{B} 2$ is not surprising because the bar pattern speed tends to seek out the local $\Omega-\kappa / 2$, which is larger in the center (also see Kormendy \& Kennicutt 2004). The pattern speed of the $\mathrm{B} 2, \Omega_{\mathrm{B} 2}$, also varies with the relative phase of the two bars; it is larger when the two bars align and smaller when they are orthogonal. We plot in Figure 3 the system in the corotating frame of the $\mathrm{B} 1$. The variations of both $A_{\mathrm{B} 2}$ and $\Omega_{\mathrm{B} 2}$ are readily visible. The variation of $\Omega_{\mathrm{B} 2}$ can be $>20 \%$ but is much less significant for $\Omega_{\mathrm{B} 1}$ (Fig. 4). Defining $\left\langle\Omega_{\mathrm{B} 2}\right\rangle\left(\left\langle\Omega_{\mathrm{B} 1}\right\rangle\right)$ as the average pattern speed of the B2 (B1) over one relative rotation, we plot in the inset of Figure 4 the phase difference between $\left\langle\Omega_{\mathrm{B} 2}\right\rangle t\left(\left\langle\Omega_{\mathrm{B} 1}\right\rangle t\right)$ and the phase of the $\mathrm{B} 2(\mathrm{~B} 1)$. The B1 is seen to rotate with a rather constant $\Omega_{\mathrm{B} 1}$, but the B2 experiences a large variation in $\Omega_{\mathrm{B} 2}$ over one relative rotation.

Figure 5 presents ellipse fits using IRAF for times when the B2 and B1 are perpendicular and at $\sim 45^{\circ}$ to each other. In both cases the phase of the $\mathrm{B} 2$ is constant to within $10^{\circ}$ and there is little sense of spirality in it. This is distinctly a nuclear bar rather than a nuclear spiral.

We measured the sizes of the two bars, for two different relative orientations at $t=340$ and 350 , as the larger radius where the bar phase deviates by more than $10^{\circ}$ from a constant value. We find a semimajor axis ratio $\simeq 0.10(\simeq 0.12)$ at $t=$ $340(t=350)$, in good agreement with the typical size ratio of local S2B systems (Erwin \& Sparke 2002). 

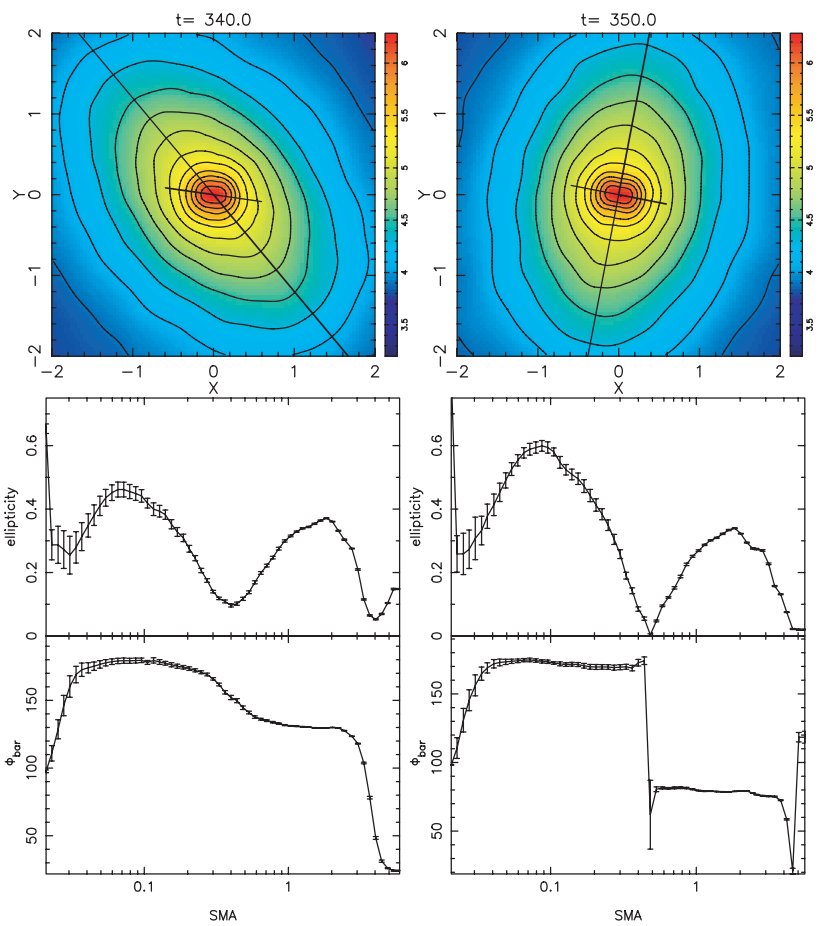

FIG. 5.-Results of ellipse fits using IRAF. Top: Snapshots of run 1 at $t=$ 340 (left) and $t=350$ (right). The two bars are at $\sim 45^{\circ}$ at $t=340$ and perpendicular at $t=350$. Middle: Ellipticity as a function of semimajor axis (SMA) of fitted ellipses. Bottom: Position angle as a function of SMA of fitted ellipses.

\section{DISCUSSION AND CONCLUSIONS}

Our self-consistent simulations of S2B systems can be compared to the models of MS00. The simulations all exhibited oscillating pattern speeds and amplitudes for one or both bars. Similarly MS00 found that the $x_{2}$ loops supporting the B2 change axis ratios and lead or trail the rigid figure of the B2 as the bars rotate. The loop orbits of MS00 were more elongated in the $\mathrm{B} 2$ region when the two bars were orthogonal than when they were parallel. The pulsating character of the self-consistent B2 in the simulations provides strong evidence that $x_{2}$ loops are the backbone of the double bars in these simulations. This behavior is also in good agreement with the earlier prediction by Louis \& Gerhard (1988) that independent rigid rotation of two bars is not possible. The $x_{2}$ loop orbits of MSO0 also suggested that $\Omega_{\mathrm{B} 2}$ would be largest when the two bars are parallel, which is also borne out by the simulations. Furthermore, MS00 were unable to find supporting $x_{2}$ orbits when the B2 extended to about $R_{c, \mathrm{~B} 2}$, while we found that $\Omega_{\mathrm{B} 2}$ had to decrease once the B1 formed and the B2 did not extend to $R_{c, \mathrm{~B} 2}$, again in good agreement with MS00. Our simulations also suggest that observationally there should be a slight excess of close-to-perpendicular double bars, as the secondary bar tends to rotate slower when two bars are perpendicular.

The main objective of this work is to create S2B systems and show how the two bars form spontaneously, interact, and evolve. Our simulations all form B2s before they form B1s. However, this is not a prediction of our model, and it occurs only because, for simplicity, we introduced our rotating pseudobulge from $t=0$. It is more likely that a pseudobulge would form after gas is driven to the center by a preexisting B1. Our pseudobulges all had rotation; B2s did not form in simulations without pseudobulge rotation. In contrast, Rautiainen \& Salo (1999) produced B2s even though their bulges were analytic.

We are able to form well-resolved, long-lived B2s in purely collisionless $\mathrm{N}$-body simulations. The nuclear bars are distinctly barred, not spiral, and reach to the center. These simulations demonstrate that B2s do not need to be gaseous. We confirm that pseudobulge rotation may be an important ingredient for the formation of double-barred galaxies (Kormendy \& Kennicutt 2004). The required degree of rotation is modest and not greater than that associated with pseudobulges (e.g., Kormendy 1982, 1993). The B2s in these simulations rotate faster than the B1s. The implications of the pulsating nature of B1s on central gas inflow are unclear at present. This new method for forming S2B models reliably and repeatedly should prove a boon to exploring their dynamics and evolution, their observational properties, their effect on gas, etc. We will report on these issues elsewhere.

We thank Jerry Sellwood and John Kormendy for helpful comments on the manuscript. V. P. D. is supported by a Brooks Prize Fellowship at the University of Washington and receives partial support from NSF ITR grant PHY-0205413.

\section{REFERENCES}

Begelman, M. C., Volonteri, M., \& Rees, M. J. 2006, MNRAS, 370, 289

Buta, R., \& Crocker, D. A. 1993, AJ, 105, 1344

Corsini, E. M., Debattista, V. P., \& Aguerri, J. A. L. 2003, ApJ, 599, L29

Davies, C. L., \& Hunter, J. H. 1997, ApJ, 484, 79

Debattista, V. P., \& Sellwood, J. A. 2000, ApJ, 543, 704

de Vaucouleurs, G. 1975, ApJS, 29, 193

Emsellem, E., Greusard, D., Combes, F., Friedli, D., Leon, S., Pécontal, E., \& Wozniak, H. 2001, A\&A, 368, 52

Englmaier, P., \& Shlosman, I. 2004, ApJ, 617, L115

Erwin, P., \& Sparke, L. S. 2002, AJ, 124, 65

Friedli, D. 1996, A\&A, 312, 761

Friedli, D., \& Martinet, L. 1993, A\&A, 277, 27

Heller, C., Shlosman, I., \& Englmaier, P. 2001, ApJ, 553, 661

Kormendy, J. 1982, ApJ, 257, 75

—. 1993, in IAU Symp. 153, Galactic Bulges, ed. H. Dejonghe \& H. J.

Habing (Dordrecht: Kluwer), 209

Kormendy, J., \& Kennicutt, R. C., Jr. 2004, ARA\&A, 42, 603

Kuijken, K., Fisher, D., \& Merrifield, M. R. 1996, MNRAS, 283, 543

Laine, S., Shlosman, I., Knapen, J. H., \& Peletier, R. F. 2002, ApJ, 567, 97

Louis, P. D., \& Gerhard, O. E. 1988, MNRAS, 233, 337

Lynden-Bell, D. 1962, MNRAS, 123, 447

Maciejewski, W., \& Sparke, L. S. 1997, ApJ, 484, L117
Maciejewski, W., \& Sparke, L. S. 2000, MNRAS, 313, 745

Maciejewski, W., Teuben, P. J., Sparke, L. S., \& Stone, J. M. 2002, MNRAS, 329,502

Moiseev, A. V., Valdés, J. R., \& Chavushyan, V. H. 2004, A\&A, 421, 433

Mulchaey, J. S., Regan, M. W., \& Kundu, A. 1997, ApJS, 110, 299

Petitpas, G. R., \& Wilson, C. D. 2002, ApJ, 575, 814

. 2004, ApJ, 603, 495

Prendergast, K. H., \& Tomer, E. 1970, AJ, 75, 674

Rautiainen, P., \& Salo, H. 1999, A\&A, 348, 737

Rautiainen, P., Salo, H., \& Laurikainen, E. 2002, MNRAS, 337, 1233

Schinnerer, E., Maciejewski, W., Scoville, N., \& Moustakas, L. A. 2002, ApJ, 575,826

Sellwood, J. A., \& Merritt, D. 1994, ApJ, 425, 530

Sellwood, J. A., \& Valluri, M. 1997, MNRAS, 287, 124

Shlosman, I., Frank, J., \& Begelman, M. C. 1989, Nature, 338, 45

Shlosman, I., \& Heller, C. H. 2002, ApJ, 565, 921

Silverman, B. W. 1986, Density Estimation for Statistics and Data Analysis (London: Chapman \& Hall)

Springel, V., Di Matteo, T., \& Hernquist, L. 2005, MNRAS, 361, 776

Toomre, A. 1981, in Structure and Evolution of Normal Galaxies, ed. S. M. Fall \& D. Lynden-Bell (Cambridge: Cambridge Univ. Press), 111 